\title{
Evaluación de usabilidad y accesibilidad de un conjunto de dispositivos interactivos denominados Puntos de Información Ciudadana
}

\section{Marta Oliva}

Universitat de Lleida

\section{Llúcia Masip}

Universitat de Lleida

\section{Toni Granollers}

Universitat de Lleida

\section{Resumen.}

Este trabajo describe el proceso y el análisis de los resultados de un proyecto de evaluación de la usabilidad y accesibilidad de un conjunto de dispositivos interactivos denominados Puntos de Información Ciudadana. Estos son unos terminales que el Ayuntamiento de Lleida ha distribuido en diferentes localizaciones estratégicas de la ciudad, con el objetivo de facilitar el uso de servicios y sistemas de información que el consistorio ofrece a sus ciudadanos. La iniciativa sirve además para acercar las TICs a todas las capas sociales y disminuir la fractura tecnológica entre la población. Los puntos clave del proyecto residen en los parámetros a considerar para realizar una evaluación a este tipo de dispositivos (evaluación estrechamente relacionada al contexto de interacción) y la forma de aplicar las metodologías de evaluación de la usabilidad y accesibilidad para este tipo de contextos.

Palabras clave: usabilidad, accesibilidad, contexto interacción, evaluación heurística

\begin{abstract}
:
This paper describes the process and the results analysis of a usability and accessibility evaluation project for a set of interactive devices called Citizen Information Points. These are specific designed interactive terminals that Lleida City Council distributed around strategic locations in the city. Its aim is enabling and facilitating the use of the information systems and services offered by the municipality to the citizens. The initiative also serves for supplying the Information and Communication Technologies (ICTs) to all levels of society and reduces the technological divide among the population. The key points of the project are the parameters to consider when evaluating this type of devices (evaluation closely related to the interaction context) and how to apply the methodologies for evaluating the usability and accessibility for such contexts.
\end{abstract}

Keywords: usability, accessibility, context interaction, heuristic evaluation 


\section{Introducción}

Desde hace varios años, el grupo de investigación en Interacción Persona-Ordenador e Integración de Datos, GRIHO, de La Universitat de Lleida, viene realizando tareas vinculadas con la usabilidad y la accesibilidad de servicios digitales dependientes del ayuntamiento de la ciudad. El vínculo entre ambas entidades es permanente y está formalizado mediante un convenio bilateral que permite establecer un conjunto de acciones mínimas a realizar anualmente.

En las primeras etapas se han cubierto necesidades vinculadas con la accesibilidad de los servicios ofrecidos por la corporación municipal y con la usabilidad de los sitios web de departamentos dependientes de alcaldía. Tras esta primera etapa, nos planteamos para el año 2009 un reto diferente. Nos propusimos evaluar la usabilidad y la accesibilidad de unos dispositivos interactivos denominados Puntos de Información Ciudadana ubicados en edificios estratégicos de Lleida. Evaluar semejantes características en esta tipología de dispositivos es completamente diferente a hacerlo para un sistema de sobremesa e incluso que hacerlo para un dispositivo móvil.

El consistorio diseñó y repartió los dispositivos en las ubicaciones definidas. Posteriormente, tras un periodo experimental de aproximadamente un año en el que los dispositivos se encuentran a disposición de todos los ciudadanos, encarga al grupo GRIHO evaluar los aspectos relacionados con la facilidad de uso y los aspectos básicos referentes al acceso para personas con discapacidad. Éstos, junto a la recomendación de no utilizar más recursos de los necesarios, constituyen los objetivos de la experiencia descrita en este artículo. En los siguientes apartados se describen brevemente cómo son los dispositivos y cómo se ha procedido para realizar la evaluación planteada.

\section{Los Puntos de Información Ciudadana}

Como se indica en el portal web de "La Paeria" (denominación local del Ayuntamiento de Lleida), los Puntos de Información Ciudadana (PIC) (ver Ilustración 1) son terminales de uso público, conectados mediante Internet a los servicios web del Ayuntamiento de Lleida y que están instalados en dependencias municipales, pudiendo ser utilizados por todos los ciudadanos y ciudadanas. Permiten acceder tanto a la información municipal como a la información propia de cada ciudadano, así como realizar todo tipo de gestiones y obtener certificados y volantes.

Todos los terminales están provistos de lectores de firma digital, tanto con tarjeta criptográfica como con el DNI electrónico o con los lápices USB de IdCat que distribuye gratuitamente el Ayuntamiento. Además, están equipados con impresoras para ofrecer mayor servicio al ciudadano, de esta forma se pueden imprimir mapas, documentos del tablón de anuncios, volantes de empadronamiento, copias de recibos de pago, etc.

\section{Metodología utilizada para la evaluación}

Tras analizar las distintas metodologías de evaluación de la usabilidad utilizadas en la disciplina de la Interacción Persona-Ordenador (IPO), y teniendo en cuenta las recomendaciones de minimización de recursos, el equipo del grupo de investigación consideró adecuado utilizar el método por inspección 
denominado Evaluación Heurística (Nielsen y Molich, 1990). Este método permite obtener resultados en poco tiempo involucrando un número pequeño de personas (Lindgaard,1994).

Dadas las características de los terminales a analizar se tuvieron que establecer el conjunto de categorías formadas por las heurísticas más apropiadas, teniendo en cuenta que el análisis no debía centrarse en los servicios ofrecidos mediante los PIC sino en la facilidad en el uso y el acceso a los terminales. Para ello se tubo en cuenta literatura relacionada con el diseño y evaluación de quioscos públicos (Maguire, 1997; Nieto, 2002, Evolucy, 2004; Gutierrez, 2007), y de diseño y evaluación en general (Granollers et al., 2005; Fundación Sidar), así como información relevante extraída de algunos blogs de Internet de contrastada referencia (facilusar.com, Usable y Accesible).

Todo ello, manteniendo de forma prioritaria las pautas establecidas en las normativas vigentes UNE139801: 2003 y UNE139802: 2003 relacionadas con la accesibilidad digital. En las subsecciones siguientes se describen las heurísticas utilizadas, agrupadas por categorías, el sistema de puntuación de la severidad establecido y el procedimiento utilizado para realizar la recogida de datos durante la evaluación.

\subsection{Heurísticas utilizadas}

Teniendo en cuenta toda la documentación relacionada y los requisitos de la evaluación de los PIC, se consideró conveniente establecer 10 categorías de heurísticas: distribución física, software del dispositivo, interacción con el dispositivo, Hardware (Pantalla), Hardware (Ratón), Hardware (Teclado), Hardware (asistencial), Hardware (Audio), indicadores y, por último, documentación y ayuda.

A continuación se describen los objetivos principales del uso de cada una de las categorías, mostrando las heurísticas aplicadas para cada una de ellas.

\subsubsection{Distribución física.}

Los objetivos principales establecidos para esta categoría consisten en velar por la visibilidad total del dispositivo para que los usuarios sean conscientes de su existencia y su ubicación facilite el acceso de cualquier persona a él, así como el fácil acceso a los dispositivos de almacenamiento externo y a la impresora. Para garantizar el cumplimiento de estos objetivos se utilizaron 6 heurísticas (véase el detalle en la siguiente tabla).

Tabla 1. Heurísticas correspondientes a la categoría de Distribución física

\begin{tabular}{|l|}
\hline 1. Distribución física \\
\hline 1.1El lugar dónde está ubicado el dispositivo PIC es visible \\
\hline 1.2 Próximo al dispositivo existen indicadores que informen de su existencia \\
\hline 1.3 Se puede acceder al PIC mediante silla de ruedas \\
\hline 1.4 La ubicación no es un lugar de paso continuo de personas \\
\hline 1.5 Los puertos de conexión de dispositivos de almacenamiento externo son de fácil acceso \\
\hline 1.6 La impresora es de fácil acceso \\
\hline
\end{tabular}

\subsubsection{Software del dispositivo.}

En cuanto a la categoría de software del dispositivo los objetivos principales consisten en cuidar la uniformidad del software en los distintos dispositivos PIC, tanto a nivel de sistema operativo como de navegador de Internet, siendo una prioridad específica del Ayuntamiento el uso de software libre. Además, se debía analizar la disponibilidad de software específico para personalizar la interfaz según las preferencias o discapacidades 
de los usuarios. Para ello se determinaron 6 heurísticas que pueden verse en siguient e tabla.

Tabla 2. Heurísticas correspondientes a la categoría de Software del dispositivo

\begin{tabular}{|l|}
\hline 2. Software del dispositivo \\
\hline 2.1 Utiliza sistema operativo Linux (en caso contrario indicar cual) \\
\hline 2.2 Versión del sistema operativo \\
\hline 2.3 Utiliza como navegador el Mozilla Firefox (en caso contrario indicar cual) \\
\hline 2.4 Versión del navegador \\
\hline 2.5 El terminal dispone de un lector de pantallas para personas con visibilidad muy baja o nula \\
\hline 2.6 El software permite personalizar la interfaz según las preferencias estándar del navegador \\
\hline
\end{tabular}

\subsubsection{Interacción con el dispositivo}

Las 5 heurísticas (Tabla 3) utilizadas para esta categoría velan por la facilidad de uso del dispositivo, considerando todos sus componentes, para todo tipo de usuario independientemente de sus características físicas, prestando especial atención al dispositivo de interacción que simula un ratón convencional. Es también objetivo de esta categoría el vigilar que el uso de la iconografía sea el adecuado.

Tabla 3. Heurísticas correspondientes a la categoría de Interacción con el dispositivo

\begin{tabular}{|l|}
\hline 3. Interacción con el dispositivo \\
\hline $\begin{array}{l}\text { 3.1 El dispositivo de interacción que simula un ratón convencional es intuitivo, de fácil } \\
\text { reconocimiento y uso }\end{array}$ \\
\hline $\begin{array}{l}\text { 3.2 La altura del terminal permite poder interactuar con él a cualquier persona (tanto si utiliza silla de } \\
\text { ruedas como si se trata de alguien muy alto o muy bajo) }\end{array}$ \\
\hline 3.3 Los iconos utilizados son comprensibles \\
\hline 3.4 El uso del dispositivo no requiere de gran esfuerzo físico \\
\hline 3.5 El uso del ratón es universal \\
\hline
\end{tabular}

\subsubsection{Hardware (Pantalla)}

El objetivo principal de esta categoría es cuidar que la visualización del contenido en la pantalla sea adecuada. Para ello, se utilizan las 4 heurísticas que se describen en la Tabla 4. Cabe destacar la importancia de que la pantalla sea ajustable a las necesidades del usuario según sus características y según las características del entorno.

Tabla 4. Heurísticas correspondientes a la categoría de Hardware (Pantalla)

\begin{tabular}{|l|}
\hline 4. Hardware (Pantalla) \\
\hline 4.1 La inclinación de la pantalla, así como la luz que en ella se refleja, es adecuada \\
\hline 4.2 Se puede ajustar el color, brillo y contraste para que se adapte a las condiciones ambientales \\
\hline 4.3 Ofrece un mecanismo para cambiar la posición manual o automáticamente \\
\hline 4.4 La pantalla no parpadea \\
\hline
\end{tabular}

\subsubsection{Hardware (Ratón)}

El ratón se ha convertido en uno de los elementos de interacción incluido en todo tipo de ordenador, a pesar de que no todos los usuarios pueden hacer uso de él con facilidad. Es por ello que las 2 heurísticas (ver Tabla 5) de esta categoría velan por la uniformidad de éste.

Tabla 5. Heurísticas correspondientes a la categoría de Hardware (Ratón)

\begin{tabular}{|l|}
\hline 5. Hardware (Ratón) \\
\hline 5.1 El ratón consta, aparte de la bola, de dos botones que simulan los de un ratón de sobremesa \\
\hline $5.2 \mathrm{Si}$ el ratón dispone de botones adicionales para su funcionamiento, se asocia fácilmente su objetivo \\
\hline
\end{tabular}

\subsubsection{Hardware (Teclado)}

El componente de entrada de datos principal es el teclado. Esta categoría tiene, por ello, el objetivo de cuidar que el teclado pueda tener un uso fácil y universal. Así, las 7 heurísticas (Tabla 6) de esta categoría indagan aspectos relacionados con el uso cómodo 
e intuitivo del teclado por parte de cualquier persona, como de la existencia de marcado táctil para facilitar el uso a personas con discapacidades visuales.

Tabla 6. Heurísticas correspondientes a la categoría de Hardware (Teclado)

\begin{tabular}{|l|}
\hline 6. Hardware (Teclado) \\
\hline 6.1 Las teclas requieren de un esfuerzo superior al habitual para ser pulsadas \\
\hline 6.2 La pulsación de las teclas transmite al usuario una sensación táctil y / o sonora \\
\hline 6.3 Las teclas disponen de marca táctil (lenguaje Braille) \\
\hline 6.4 Las teclas se perciben de forma táctil sin que este hecho provoque una activación de las mismas \\
\hline 6.5 El color no debe ser la única manera de diferenciar las teclas \\
\hline 6.6 Cada grupo funcional de teclas utiliza un color distintivo \\
\hline 6.7 Las etiquetas de las teclas son fácilmente legibles \\
\hline
\end{tabular}

\subsubsection{Hardware (asistencial)}

Cuando un dispositivo quiere ser universal es imprescindible que disponga de los correspondientes elementos asistenciales. Éste es el objetivo de la única heurística (Tabla 7) perteneciente a esta categoría.

Tabla 7. Heurísticas correspondientes a la categoría de Hardware (asistencial)

\section{Hardware (asistencial)}

7.1 Si dispone de técnicas asistenciales incorpora los dispositivos HW necesarios para su utilización

\subsubsection{Hardware (Audio)}

El objetivo de esta categoría es velar por el buen uso de los componentes audio del terminal, en el momento en que estos deban producir algún tipo de sonido. Además, resulta imprescindible que todos los PIC sean uniformes en cuanto a la emisión de información auditiva. A esta categoría le corresponden las 4 heurísticas mostradas en la Tabla 8.

Tabla 8. Heurísticas correspondientes a la categoría de Hardware (Audio)
\begin{tabular}{|l|l|}
\hline 8 Hardware (Audio) \\
\hline 8.1 El dispositivo PIC emite información auditiva \\
\hline 8.2 Si emite algún sonido, muestra la información que se pretende transmitir a través del sonido de \\
forma visual en la pantalla \\
\hline 8.3 Si emite algún sonido, el volumen de éste es el idóneo \\
\hline 8.4 Si emite algún sonido, es posible regular el volumen \\
\hline
\end{tabular}

\subsubsection{Indicadores}

Todos los elementos que componen el terminal y que ofrecen un servicio determinado a las personas deben estar adecuadamente indicados. A ello se dedican las dos heurísticas (Tabla 9) pertenecientes a esta categoría.

Tabla 9. Heurísticas correspondientes a la categoría de Indicadores

\begin{tabular}{|l|}
\hline 9. Indicadores \\
\hline 9.1 El indicador de dónde está la impresora es fácilmente visible \\
\hline 9.2 El indicador de dónde se introduce el certificado digital y / o la tarjeta electrónica es fácilmente \\
visible. \\
\hline
\end{tabular}

\subsubsection{Documentación y ayuda.}

Esta última categoría tiene el objetivo principal de vigilar que existan elementos de ayuda, fácilmente entendibles, que guíen a las personas en el uso del terminal (ver las heurísticas en la Tabla 10). 
Tabla 10. Heurísticas correspondientes a la categoría de Documentación y ayuda

\begin{tabular}{|l|}
\hline 10. Documentación y ayuda \\
\hline 10.1 El dispositivo da mensajes de ayuda para su utilización \\
\hline 10.2 Si existen carteles o documentación de ayuda, son comprensibles \\
\hline $\begin{array}{l}\text { 10.3 Existe algún recurso adicional donde encontrar información o ayuda para utilizar el PIC y éste } \\
\text { aparece indicado en el dispositivo. }\end{array}$ \\
\hline
\end{tabular}

\subsection{Sistema de puntuación de la severidad}

Una vez determinadas las heurísticas, se decidió el sistema más conveniente para puntuar la severidad de cada una de ellas. Aparecen en la literatura muchas aproximaciones sobre cómo cuantificar los resultados de una evaluación heurística, aunque no en todas las experiencias se utilizan los tres factores (frecuencia, impacto y persistencia) de puntuación de la severidad propuestos por Nielsen. Además, cabe destacar las distintas escalas de puntuación utilizadas, por ejemplo, en (González, 2009) se utiliza la escala 0-4 propuesta por Nielsen, en (Marcos, 2006) la escala utilizada es 02, en (Sutcliffe, 2004) se utiliza una escala más amplia, de 1 a 7.

En este proyecto, se consideró únicamente el factor impacto y se optó por dos tipos de puntuación. Por un lado, una respuesta binaria (sí o no), para plasmar con facilidad el cumplimiento o no de cada heurística y, por otro, la respuesta binaria se complementó con un color de referencia emulando la metáfora del semáforo (forma visual de representar los valores posibles de un argumento con tres estados posibles). Mediante esta metáfora se utilizó el verde para indicar que la heurística tratada "No supone ningún problema", naranja para cuando "es importante resaltar o merece tenerlo en cuenta” y rojo para determinar "carencias y errores evidentes". De cara a puntuar el resultado de la evaluación de cada heurística para extraer finalmente un resultado cuantitativo general de grado de usabilidad de la web, se hizo una transformación de cada color a un valor numérico en la escala 0-1.

Esta forma de proceder surge de experiencias previas de proyectos de transferencia de conocimiento y de tecnología que el grupo ha realizado a diversas entidades y empresas. Utilizar la metáfora del semáforo, al ser comprensible por prácticamente todas las personas, facilita la representación y la síntesis de la información cuando se pretende que el receptor se centre en el mensaje y no en la forma de representarlo.

Para cada heurística, además, se estableció la posibilidad de escribir observaciones específicas altamente necesarias para evaluaciones cualitativas. (Pinelle, 2008; Marcos, 2006; González, 2009) son ejemplos en los que se refleja lo comúnmente utilizadas que son las evaluaciones cualitativas en la obtención de resultados en evaluaciones heurísticas. Mediante ambas puntuaciones (la binaria y la de colores) y las observaciones realizadas, se consigue obtener unos resultados objetivos y aproximados a la realidad de cada PIC.

\subsection{Procedimiento de evaluación y recogida de datos}

El proceso de evaluación y recogida de datos fue realizado por tres evaluadoras expertas en evaluación de usabilidad de sistemas interactivos y consistió en los siguientes pasos:

1. Cada evaluadora acudió al lugar donde está ubicado cada PIC de forma totalmente individualizada para que la evaluación de una no condicionara los resultados de la evaluación de la otra. Además, cada evaluadora realizó la prueba en un horario distinto para que los elementos contextuales de cada PIC (como intensidad de luz, 
volumen de personas hablando,...) se vieran reflejados en los resultados de la evaluación.

2. Durante la evaluación, cada heurística se analizó cuidadosamente y se anotaron las puntuaciones correspondientes siguiendo la convención de los dos tipos de puntuación previamente descritos. En la Tabla 11 se incluye una pequeña muestra del formulario utilizado para anotar las puntuaciones durante el análisis a un PIC determinado (se evaluaron de forma individual cada uno de los 11 PIC existentes en la ciudad de Lleida). Cuando se consideró oportuno, se tomaron fotografías que permitieran ilustrar los problemas de forma más sencilla y precisa al elaborar el informe de resultados. Cada evaluadora dedicaba aproximadamente entre 30 y 45 minutos en la evaluación de cada dispositivo.

Tabla 11. Ejemplo de Formulario utilizado para la puntuación de las heurísticas en cada evaluación

\begin{tabular}{|l|l|l|l|l|}
\cline { 2 - 4 } \multicolumn{1}{c|}{} & Si & No & $\begin{array}{c}\text { Color } \\
\text { semáforo }\end{array}$ & Observaciones \\
\hline $\begin{array}{l}\text { 1.Distribución física } \\
\text { visible }\end{array}$ & & & & \\
\hline $\begin{array}{l}\text { 1.2 Próximo al dispositivo existen indicadores que } \\
\text { informan de su existencia }\end{array}$ & & & & \\
\hline
\end{tabular}

3. Posteriormente, y tal cómo el método establece, se realizó una reunión entre las tres evaluadoras para poner en común los resultados individuales. En esta reunión se presto una atención especial a aquellos resultados no coincidentes de cada una de las heurísticas y se procuró llegar a un consenso para cada PIC. Este análisis de síntesis permitió elaborar el informe final de resultados.

\section{Resultados de la evaluación}

Como es habitual en toda evaluación heurística, gracias a la evaluación individual de cada dispositivo y a la reunión de discusión de las puntuaciones no coincidentes, se obtuvieron resultados cualitativos. Por otro lado, haciendo uso del porcentaje de cumplimiento de cada heurística, se hizo un pequeño esfuerzo para conseguir resultados cuantitativos. El objetivo de los resultados cuantitativos es poder establecer un orden de prioridad/urgencia a la hora de aplicar propuestas de mejora, Así pues, a continuación se detallan ambos resultados, los cualitativos y los cuantitativos.

\subsection{Resultados cualitativos}

En esta sección se presentan los resultados cualitativos obtenidos de la evaluación heurística, especificándolos por categorías. Podrá observarse que algunos de los aspectos detectados requieren de un mínimo esfuerzo para ser mejorados, teniendo en cuenta que suele tratarse de detalles que a simple vista pueden parecer superficiales pero que resultan de mucha ayuda para las personas que quieran utilizar los terminales.

Así pues, para cada categoría (mediante las Tablas de la 12 a la 19) se citan las heurísticas que no se cumplen en ningún PIC, las observaciones realizadas por las evaluadoras y una posible propuesta de mejora a aplicar para solucionar el problema y cumplir con cada heurística. 
Tabla 12. Heurísticas que no se cumplen de la categoría: Distribución Física.

\begin{tabular}{|l|l|}
\hline \multicolumn{2}{|l|}{ Categoría: Distribución física } \\
\hline Pauta: 1.2 Próximo al dispositivo existen indicadores que informan de su existencia \\
\hline Observaciones: & $\begin{array}{l}\text { No existe ningún indicador y/o cartel que informe sobre qué es el PIC y para qué } \\
\text { sirve. }\end{array}$ \\
\hline $\begin{array}{l}\text { Propuesta de } \\
\text { mejora: }\end{array}$ & $\begin{array}{l}\text { Incorporar carteles explicativos de qué es el dispositivo, para qué sirve y dónde } \\
\text { dirigirse en caso de incidencia. Se podría completar con una campaña de marketing } \\
\text { para fomentar o popularizar su uso. }\end{array}$ \\
\hline
\end{tabular}

Tabla 13. Heurísticas que no se cumplen de la categoría: Software del dispositivo.

Categoría: Software del dispositivo

2.5 El terminal dispone de un lector de pantallas para personas con visibilidad muy baja o nula

Observaciones: $\quad$ Si una persona ciega o con baja visibilidad decide utilizar el dispositivo, este no está adaptado para que lo haga cómodamente.

\begin{tabular}{ll|l} 
Propuesta de & Añadir código Braille al teclado.
\end{tabular}

mejora:

2.6 El software permite personalizar la interfaz según las preferencias estándar del navegador.

Observaciones: $\quad$ El navegador permite cambiar algunas de las propiedades de visualización de la pantalla pero son limitadas y escasas.

\begin{tabular}{l|l} 
Mejora: & Proveer al usuario de las propiedades necesarias para que se pueda configurar el
\end{tabular} dispositivo cubriendo sus necesidades.

Tabla 14. Heurísticas que no se cumplen de la categoría: Interacción con el dispositivo.

Categoría: Interacción con el dispositivo

3.1 El dispositivo de interacción que simula un ratón convencional es intuitivo, de fácil reconocimiento y uso.

\begin{tabular}{|l|l|l|l|}
\hline Observaciones: & $\begin{array}{l}\text { Se reconoce el dispositivo que representa el ratón pero posiblemente los usuarios más } \\
\text { noveles tengan dificultades para interaccionar con él. }\end{array}$ \\
$\begin{array}{l}\text { Propuesta } \\
\text { mejora: }\end{array}$ & $\begin{array}{l}\text { Modificar el diseño actual del ratón (bola } \\
\text { joystick) por algún otro modelo de } \\
\text { interacción como puede ser un touchpad. }\end{array}$ & Figura 2. Teclado y ratón actuales \\
\hline
\end{tabular}

3.3 Los iconos utilizados son comprensibles

Observaciones: $\quad$ La pantalla de entrada al dispositivo muestra el icono de Mozilla Firefox y del PinClauer (Certificado digital que ofrece el Ayuntamiento de Lleida). Ambos iconos, a parte de no llevar asociada ninguna descripción, no son representativos de sus funciones.

\begin{tabular}{l|l} 
Mejora: & Aprovechar todo el espacio de la pantalla inicial para mejorar los iconos utilizados y
\end{tabular} acompañarlos de descripciones que definan sus funcionalidades.

3.5 El uso del ratón es universal

Observaciones: $\quad$ En todos los dispositivos el ratón está situado a la parte derecha del teclado por lo que dificulta la interacción a las personas zurdas.

\begin{tabular}{l|l} 
Mejora: & Situar el ratón en la parte inferior del teclado de modo que se quede en el centro de la
\end{tabular} tabla de interacción.

Tabla 15. Heurísticas que no se cumplen de la categoría: Hardware (Pantalla).

Categoría: Hardware (Pantalla)

4.2 Se puede ajustar el color, brillo y contraste para que se adapte a las condiciones ambientales

Los dispositivos no ofrecen la posibilidad de adaptar la presentación en pantalla para

Observaciones: $\quad$ poder visualizar mejor la información de acuerdo con las características del contexto donde se sitúa.

\begin{tabular}{ll|l} 
Propuesta de & Situar el dispositivo en un lugar donde sea difícil que se refleje la luz en la pantalla.
\end{tabular} mejora:

4.3 Ofrece un mecanismo para cambiar la posición manual o automáticamente

Observaciones: $\quad$ No es posible ajustar la inclinación de la pantalla para que todos los usuarios se la sitúen en el ángulo que necesiten.

Mejora: $\quad$ Modificar el diseño de la pantalla para que sea reclinable/ajustable a las condiciones específicas de cada usuario. 
Tabla 16. Heurísticas que no se cumplen de la categoría: Hardware (Teclado).

Categoría: Hardware (Teclado)

Pauta: 6.3 Las teclas disponen de marca táctil (lenguaje Braille)

\begin{tabular}{l|ll}
\hline Observaciones: & El teclado no incluye el lenguaje Braille para las personas con discapacidades visuales
\end{tabular}

\begin{tabular}{|l|l}
\hline Propuesta de & Incorporar teclados con descripción Braille en las teclas.
\end{tabular}

mejora:

Tabla 17. Heurísticas que no se cumplen de la categoría: Hardware (asistencial).

Categoría: Hardware (asistencial)

Pauta: 7.1 Si dispone de técnicas asistenciales incorpora los dispositivos HW necesarios para su utilización

\begin{tabular}{|l|l|}
\hline Observaciones: & $\begin{array}{l}\text { Ningún PIC dispone de ninguna técnica asistencial para facilitar el uso de estos a } \\
\text { personas que sufren algún tipo de discapacidad. }\end{array}$ \\
\hline $\begin{array}{l}\text { Propuesta de } \\
\text { mejora: }\end{array}$ & $\begin{array}{l}\text { Adaptar el dispositivo para que cualquier persona, sufra o no algún tipo de } \\
\text { discapacidad, pueda utilizar el dispositivo sin ningún tipo de dificultad. }\end{array}$ \\
\hline
\end{tabular}

Tabla 18. Heurísticas que no se cumplen de la categoría: Hardware (audio).

Categoría: Hardware (audio)

Pauta: 8.1 El dispositivo PIC emite información auditiva

Observaciones: $\quad$ Ningún PIC emite información auditiva.

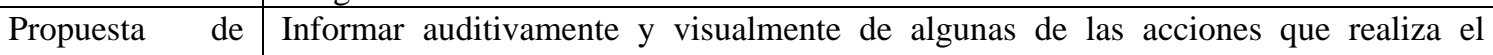
mejora: $\quad$ usuario.

Tabla 19. Heurísticas que no se cumplen de la categoría: Documentación y ayuda.

Categoría: Documentación y ayuda

Pauta: 10.1 El dispositivo da mensajes de ayuda para su utilización

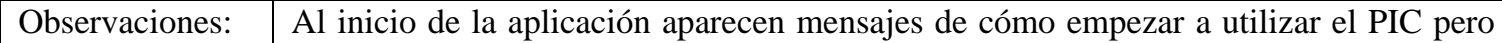
son insuficientes para poder realizar cualquier tarea con el dispositivo.

Propuesta de Ampliar esta ayuda más allá de la pantalla de inicio y, sobre todo, que la ayuda sea mejora: $\quad$ comprensible para el usuario que esté utilizando el PIC.

Aparte de los problemas encontrados en el cumplimiento de cada una de las heurísticas, en la reunión post-evaluación aparecieron los siguientes aspectos/mejoras a tener en cuenta en los resultados finales de la experiencia:

- Debido a que la situación de dichos dispositivos es un lugar público, es necesario tener en cuenta el flujo de gente que transita alrededor del PIC para asegurar al usuario tranquilidad y seguridad a la hora de utilizarlo.

- En algunas ocasiones, los indicadores de situación tanto de la impresora como del dispositivo de certificado digital pasan desapercibidos. Sería preciso colocar dichos adhesivos en un lugar vistoso para el usuario y que fueran ellos en si también vistosos.

- Los documentos impresos no quedan depositados en la bandeja diseñada para tal uso, si no que se caen al suelo. Además, resulta imposible cancelar una impresión una vez confirmada. La Ilustración 3 muestra claramente la situación descrita.

- Existen teclas cuyo significado es ambiguo: ALT, DEL, CAP LOCK, ftp, http, www, .com, :) , :) entre otras.

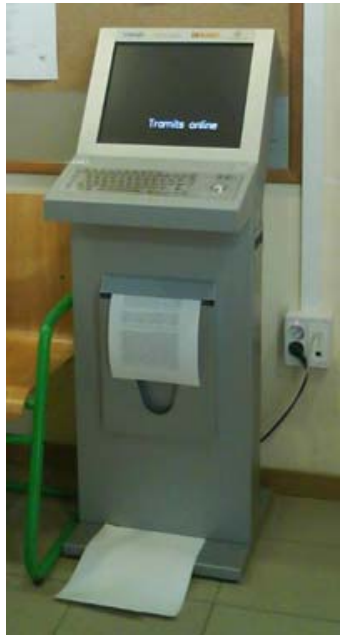

Figura 3. Imagen del terminal mientras se imprime un documento

- La velocidad de procesamiento de los dispositivos en general es lenta. Principalmente al inicio, cuando se decide abrir el navegador tarda mucho y no informa al usuario del estado del sistema por lo que en alguna ocasión se puede pensar que el dispositivo no responde. 


\subsection{Resultados cuantitativos}

Los resultados cuantitativos que se presentan de las evaluaciones realizadas son una aproximación a los resultados reales ya que científicamente se desconoce la fórmula que se puede aplicar en los resultados de una evaluación heurística para obtener un grado o nivel o representación numérica de usabilidad. El valor que aparece en los resultados obtenidos permite establecer un orden de prioridad/urgencia en la aplicación de las propuestas de mejora que se sugieren..

Así pues, la puntuación cuantitativa que se ha considerado para cada heurística viene dada por el porcentaje de cumplimiento que se calcula teniendo en cuenta los siguientes parámetros:

1. La puntuación de cada heurística (S): (0: si se cumple; 0.5: si se cumple pero es mejorable; 1: es un error que necesita solucionarse).

2. La puntuación de la categoría en la peor casuística (P): suma de la puntuación de todas las heurísticas en el peor de los casos (si todas estuvieran puntuadas con un 1).

Con estas dos variables se consigue la siguiente fórmula que representa el porcentaje de cumplimiento (PC):

$$
P C=\frac{\sum S}{P} * 100
$$

Además, para conseguir unos resultados objetivos y reales por categorías de heurísticas no se han considerado todas aquellas heurísticas que, por motivos ajenos a la voluntad de las evaluadoras, no han podido evaluarse por ninguna de ellas.

Por lo tanto las siguientes heurísticas no se han considerado en el cálculo de la puntuación final:

1. Todas las heurísticas de la categoría de Software del dispositivo.

2. De la categoría 3 (Interacción con el dispositivo), la heurística 3.3.

3. De la categoría Hardware (Pantalla), la heurística 4.2.

4. De la categoría Documentación y ayuda, las heurísticas 10.1 i 10.2.

De acuerdo con el porcentaje de cumplimiento obtenido por el conjunto de heurísticas de cada categoría, se puede establecer el siguiente ranquin por categorías de más a menos usables y accesibles:

1. Hardware (Ratón) $\rightarrow$ 93,18\% de Porcentaje de Cumplimiento (PC).

2. Documentación y ayuda $\rightarrow 90,91 \%$ de PC.

3. Hardware (Teclado) $\rightarrow 85,71 \%$ de PC.

4. Distribución física $\rightarrow 68,94 \%$ de PC.

5. Interacción con el dispositivo $\rightarrow 62,5 \%$ de PC.

6. Hardware (pantalla) $\rightarrow 54,54 \%$ de PC.

7. Indicadores $\rightarrow 50 \%$ de PC.

8. Hardware y hardware (áudio) $\rightarrow 0 \%$ de PC. 


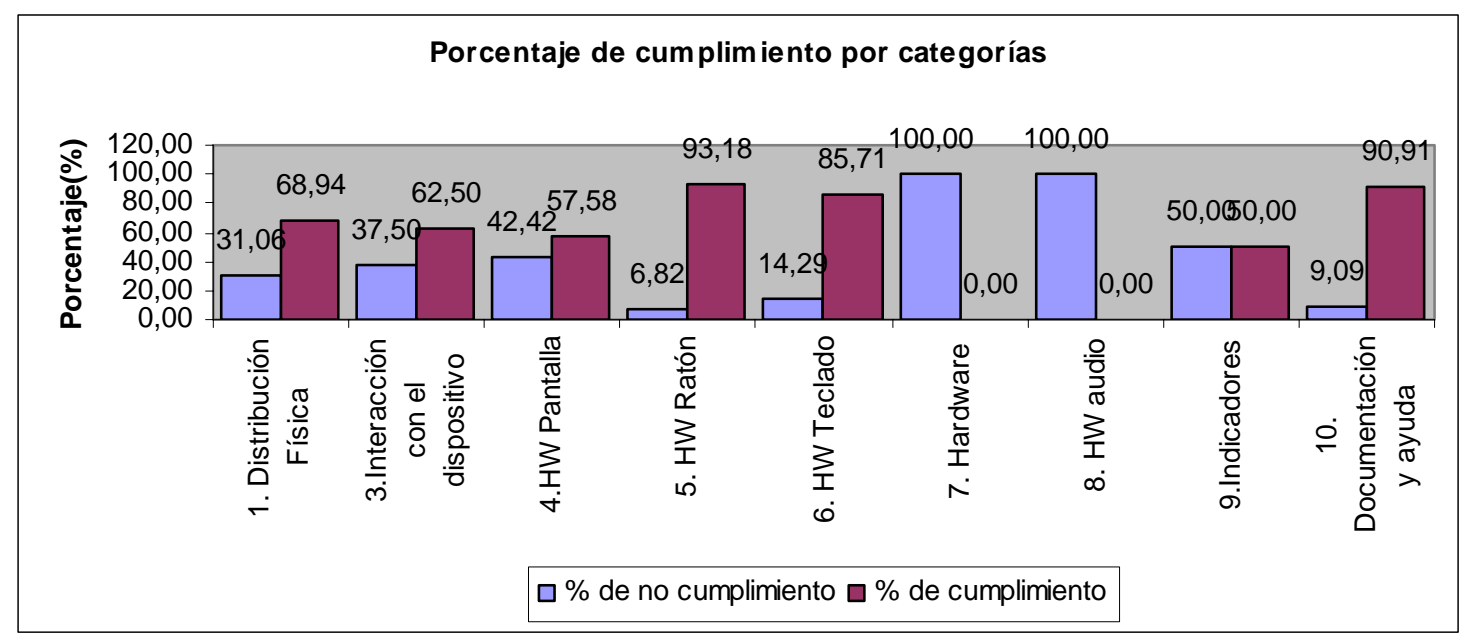

Figura 4. Gráfico de porcentajes de cumplimiento por categorías

La anterior figura recoge un gráfico con los porcentajes tanto de cumplimiento como de no cumplimiento de cada categoría de heurísticas.

\section{Conclusiones}

Este artículo contiene la descripción del proceso llevado a cabo con el fin de realizar una evaluación de la usabilidad y accesibilidad de un conjunto de dispositivos interactivos denominados Puntos de Información Ciudadana, que el Ayuntamiento de Lleida puso a disposición de los ciudadanos. El artículo ha permitido mostrar cómo con un mínimo de recursos se puede aplicar una evaluación heurística, mediante la cual se permite detectar un porcentaje elevado de problemas de usabilidad y/o de accesibilidad.

Cabe destacar que los elementos objeto de evaluación eran distintos en tipología a los habituales sistemas de sobremesa, web o portales móviles y que por ello era necesario realizar las evaluaciones de forma totalmente estrecha al contexto de la interacción.

Así pues, uno de los aspectos cruciales de la evaluación fue la elección de las heurísticas (en las distintas categorías) que podían ayudar a conseguir resultados más relevantes en el análisis. Otro de los aspectos a destacar, en beneficio del receptor de las conclusiones del análisis realizado, es el uso de resultados tanto cualitativos como cuantitativos, de forma que se incluyan propuestas de mejora y, a la vez, elementos mediante los que establecer prioridades a la hora de aplicar dichas propuestas.

\section{Referencias}

Evolucy (2004). Evaluación heurística de un quiosco de autoservicio de fotografía digital: caso práctico. Disponible en:

http://www.evolucy.com/esp/projects/evaluacion_heuristica_kiosk.html (8-7-2009)

facilusar.com. http://facilusar.blogspot.com/ (19/09/2008)

Fundación Sidar. Principios del Diseño Universal o Diseño para Todos. http://www.sidar.org/recur/desdi/usable/dudt.php (19/09/2008)

González, Marta; Masip, Llúcia; Granollers, Antoni; Oliva, Marta (2009). Quantitative analysis in a heuristic evaluation experiment. // Advances in Engineering Software. 40:12 (December 2009) 1271-1278.

Granollers, Toni; Lorés, Jesús; Cañas, José Juan. (2005). Diseño de sistemas interactivos centrados en el usuario. Barcelona: Editorial UOC. ISBN: 84-9788-320-9 
Gutiérrez, Malisa (2007).”¡Larga vida de los cajeros!”. Revista Faz. Noviembre del 2007 número 1. http://www.revistafaz.org/numero1/cajeros.pdf (19/09/2008)

La Paeria - Ayuntamiento de Lleida. Los puntos de atención ciudadana del ayuntamiento de Lleida. http://www.paeria.cat/pic/es/ (15-7-2009)

Lindgaard, G. (1994). Usability Testing and System Evaluation: A Guide for Designing Useful Computer Systems. London,U.K: Chapman and Hall. ISBN 0-412-46100-5

Maguire, M.C. (1997). A Review of User-Interface Design Guidelines for Public Information Kiosk Systems. // Stephanidis, C; Carbonell, N. (editors). Electronic Proceedings of the 3rd ERCIM Workshop on "User Interfaces for All". http://ui4all.ics.forth.gr/UI4ALL-97/maguire.pdf (15/09/2008)

Marcos, Mari-Carmen; et al. (2006). Evaluación de la usabilidad en sistemas de información terminológicos online [en línea]. "Hipertext.net", núm. 4. http://www.hipertext.net (26-10-2009)

Nielsen J. Severity Ratings for Usability Problems.

http://www.useit.com/papers/heuristic/severityrating.html (26-10-2009)

Nielsen, Jakob; Molich, Rolf (1990). Heuristic evaluation of user interfaces. // Carrasco, Jane; Whiteside, John (eds.) Proceedings of the ACM CHI 90 Human Factors in Computing Systems Conference 1990, Seattle, Washington,USA:ACM Press. 249-256.

Nieto, Miquel (2002). La usabilidad de los terminales táctiles. Evolucy. http://www.evolucy.com/esp/columns/20020515_usabilidad_tactil.html (15-7-2009)

Pinelle, David; Wong, Nelson; Stach, Tadeusz (2008). Heuristic evaluation for games: Usability principles for video Game Design. // Proceedings of ACM CHI 2008 Conference on Human Factors in Computing Systems, Florence, Italy, April 5-10, 2008 New York, NY,USA:ACM Press 1453-1462

Sutcliffe, Alistair; Gault, Brian (2004). Heuristic evaluation of virtual reality applications. // Interacting with Computers. 16 (2004) 831-849.

UNE139801 (2003). Aplicaciones informáticas para personas con discapacidad. Requisitos de accesibilidad al ordenador. Hardware. AENOR. Depósito legal: M 42219:2003.

UNE139802 (2003). Aplicaciones informáticas para personas con discapacidad. Requisitos de accesibilidad al ordenador. Software. AENOR. Depósito legal: M 42220:2003.

Usable y Accesible. http://olgacarreras.blogspot.com/ (19/09/2008)

Recibido: 2009-10-28. Aceptado: 2010-06-17

Scire. 16:1 (2010) 35-46. ISSN 1135-3716. 